\title{
Diverse Application of ECC Designed with Ground Granulated Blast Furnace Slag
}

\author{
Jeong-Su Kim ${ }^{1)}$, Yun-Yong $\mathrm{Kim}^{2) *}$, and Jin-Keun $\mathrm{Kim}^{3)}$
}

(Received November 15, 2006, Accepted December 30, 2006)

\begin{abstract}
In the recent design of high ductile engineered cementitious composites (ECC), optimizing both processing and mechanical properties for specific applications is critical. This study employs a method to develop useful ECC produced with slag particles (slag-ECC) in the field, which possesses different fluid properties to facilitate diverse types of processing (i.e., self-consolidating or spray processing). Control of rheological modulation was regarded as a key factor to allow the performance of the desired processing while retaining the ductile material properties. To control the rheological properties of the composite, the basic slag-ECC composition was initially obtained, determined based on micromechanics and steady-state cracking theory. The stability and consequent viscosity of the suspensions were then mediated by optimizing the dosage of the chemical and mineral admixtures. The rheological properties altered through this approach were revealed to be effective in obtaining ECC-hardened properties, represented by pseudo strain-hardening behavior in uniaxial tension, allowing the readily achievement of the desired function of the fresh ECC.
\end{abstract}

Keywords: ECC, slag particles, spraying, self-consolidating, rheology, chemical and mineral admixtures

\section{Introduction}

The design of materials to achieve diverse functions as specified by applications in terms of material properties and processibility is highly desirable. One example is the recently developed ultraductile engineered cementitious composites (ECCs). ${ }^{1,2}$ ECC is a micromechanically designed cementitious composite that exhibits extreme tensile strain capacity (typically more than $2 \%$ ) while using a moderate amount of fiber. Recently, a variety of applications of this material, ranging from repair and retrofit of structures to cast-in-place structures to pre-cast structural elements where high ductility was required, have been developed. ${ }^{1}$

To achieve moderately high compressive strength, targeting $50 \mathrm{MPa}$, the approach of adding ground granulated blast furnace slag (slag) has been employed. In this paper, the influence of the addition of slag particles on the matrix and interface properties that determine the strain-hardening behavior is studied. Based on this micromechanical study, the optimized range of the waterbinder ratio was obtained for the composite (ECC produced with slag particles, slag-ECC hereafter). Within this predetermined range of matrix composition, the dosage of chemical admixtures was modulated in order to develop diverse types of processing

${ }^{11} \mathrm{KCI}$ member, Dept. of Civil and Environmental Engineering, Korea Advanced Institute of Science and Technology, Daejeon 305-701, Korea.

${ }^{2)} \mathrm{KCI}$ member, Dept. of Civil Engineering, Chungnam National University, Daejeon 305-764 Korea. E-mail :yunkim@cnu.ac.kr

${ }^{3)}$ KCI member, Dept. of Civil and Environmental Engineering, Korea Advanced Institute of Science and Technology, Daejeon 305-701 Korea.

Copyright (c) 2007, Korea Concrete Institute. All rights reserved, including the making of copies without the written permission of the copyright proprietors. (i.e., self-consolidating and spraying processing), while embodying the ductile performance of ECC.

\section{Design framework}

\subsection{Micromechanical design}

The micromechanical design utilized herein is mainly focused on achieving strain-hardening in uniaxial tension, as the tensile ductility can be representative of the material ductility. A fundamental requirement for strain-hardening is the occurrence of steady state cracking, which requires the crack tip toughness to be less than the complementary energy calculated from the bridging stress vs. crack opening curve, as illustrated in Fig. $1{ }^{2}$

$$
\begin{aligned}
& J_{t i p} \leq \sigma_{0} \delta_{0}-\int_{0}^{\delta_{0}} \sigma(\delta) d \delta=J_{b}^{\prime} \\
& J_{t i p}=\frac{K_{m}^{2}}{E_{c}}
\end{aligned}
$$

where, $\sigma_{0}$ is the maximum bridging stress corresponding to the opening $\delta_{0} . K_{m}$ and $E_{c}$ are the fracture composite elastic moduli. Equation (1) is obtained by considering the balance of energy changes during the extension of a steady state flat crack. Another condition for strain-hardening is that the tensile first crack strength $\sigma_{f c}$ must not exceed the maximum bridging stress $\sigma_{0}$,

$$
\sigma_{f c}<\sigma_{o}
$$

where, $\sigma_{f c}$ is determined by the maximum flaw size max, $a_{0}$, and the matrix fracture toughness $K_{m}$. Satisfaction of Eqs. (1) and (3) is necessary to achieve ECC behavior, otherwise normal tensile 


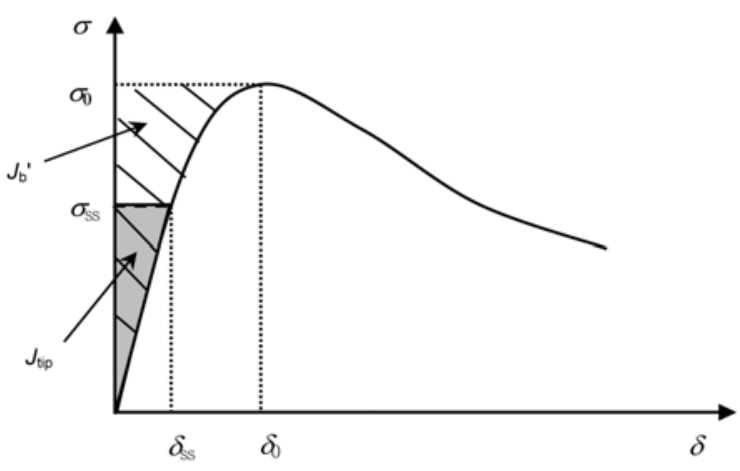

Fig. 1 A typical $\sigma(\delta)$ curve for a strain hardening composite. The hatched area represents the complementary energy $J_{b}{ }^{\prime}$, and the shaded area represents the crack tip toughness $J_{\text {tip }}$.

softening FRC behavior results.

Details of these micromechanical analyses can be found in $\mathrm{Li}$ et al. ${ }^{3}$ Satisfaction of Eqs. (1) and (3) is necessary to achieve the high ductile behavior represented by the strain-hardening behavior in uniaxial tension.

\subsection{Use of slag particles}

To achieve a moderately high compressive strength (50 MPa at 28 days) and to improve fiber dispersion, a method incorporating ground granulated blast furnace slag (slag) into cement particles is employed. A wealth of research has been conducted to improve the rheology and mechanical properties using slag particles, and it has been reported that slag can contribute to increase the flowability in a fresh state. In addition, the use of mineral admixtures (i.e., slag particles) is also desirable for economical (due to the lower cement requirement) and environ-mental considerations. The lower cement requirement also leads to a reduction in the amount of carbon dioxide generated by the production of cement, while slag particles are by-product materials ordinarily produced from metallurgical processes. Thus, there is a double environmental benefit from the use of slag particles.

For these reasons, the influences of the addition of slag particles on the matrix and interface properties that determine the strainhardening behavior have been studied. It is likely that the addition of slag particles will decrease the potential of high ductility, as the addition of slag in general results in an overall decrease in the water-binder ratio, leading to increases in $J_{t i p}$ and $\sigma_{f c}$ as well. According to the micromechanical model for steady state cracking, which is essential for achieving strain-hardening behavior, a high $J_{t i p}$ value reduces the margin for the development of multiple cracking in terms of the toughness ratio $\left(J_{b}{ }^{\prime} / J_{t i p}\right)$. Therefore, the fiber bridging behavior was initially analyzed based on the micromechanical principle, and then the range of mixture composition suitable for achieving the slag-ECC properties was determined.

Although slag particles have the side-effect to decrease, the potential of saturated multiple cracking, the use of slag is expected to provide benefits in terms of improving the fiber dispersion. It is well known that fiber dispersion is one of the key factors in achieving a sufficient mechanical performance of fiber-reinforced cementitious materials. The expected enhancement of fiber dispersion is mainly incorporated with enhanced workability attributed to the electrical double layer formed by the oxidized grain surface. Slag is a vitreous material and the surface of this particle has a polarity due to the broken Si-O bonds. The fresh behavior of slag-ECC should be positively influenced by the interactions between the cement particles and slag particles. It has been found in a previous study ${ }^{5}$ that vitreous slag particles can adsorb superplasticizer molecules and form an electrical double layer on their surfaces, thus producing a dispersion effect in the paste. This will provide a driving force to mortar matrix flowing, resulting in good fiber dispersion. The dispersion effect of slag particles should not be considered to be a micro-filling effect, as the size of the slag particles is fairly similar to that of cement particles. The dispersion effect of slag particles is related to dispersion of water molecules, but not to fiber dispersion. Other experimental results confirmed that the dispersion effect was more effective in enhancing the fluidity of slag-ECC, and was related to the vitreous phase. $^{6}$

\subsection{Rheological control by the use of admixtures}

To develop diverse processibility, the ECC fluidity was modified by optimizing the dosage of chemical admixtures. In the development of a sprayable version of slag-ECC, the focus was on modulating the dosage of chemical admixtures with the assistance of reactive mineral particles (CA), an action that provides desirable adhesion and cohesion after spraying. First, to achieve a moderate workability suitable for pumping, a proper concentration of chemical admixtures to disperse cement particles was determined. Reactive particles (CA) were then introduced to enhance the viscosity of fresh mortar over time. Based on a previous study, ${ }^{2}$ the CA dosage was optimized within $5 \%$ of the cement weight, as an overdose of reactive particles can be detrimental in achieving satisfactory pumpability, leading to an undesirable quick setting of the composite.

In contrast, self-consolidating performance is achieved by high deformability, good cohesiveness between material ingredients, and excellent consistency of the fluidity over time. A previous study $^{7}$ has demonstrated that the rheological design, which determined the optimal dosage of the chemical admixtures, is a powerful tool to modify the fluid properties of a fresh cementitious mixture.

\section{Experimental programs}

\subsection{Materials}

ECC is essentially composed of a common mortar matrix and polymer fibers. In this study, a PVA fiber (K. Co. Ltd., REC15, fiber length $=12 \mathrm{~mm}$, Japan) was used as the reinforcing fiber. Type I ordinary Portland cement (C, S. Co., average particle diameter $=11.7 \pm 4.8 \mathrm{~mm}$, Korea), silica sand (S, S. H. Silica Co., average particle diameter $=130 \pm 38 \mathrm{~mm}$, Korea), ground granulated blast furnace slag (Slag, B. M. Co., average particle diameter $=11 \pm 3$ $\mathrm{mm}$, Korea) and alumina cement (CA, U. Co., average particle diameter $=5 \pm 1 \mathrm{~mm}$, Korea) were used as the major ingredients in the matrix. Chemical admixtures comprised of high-range waterreducing admixture (HRW, A. Co., Korea) and hydroxypropylmethylcellulose (HPMC, A. Co., Korea) were used to modify the fluid properties. 


\subsection{Tests for obtaining micromechanical properties}

The appropriate composition of ingredient (cement, sand, water and chemicals) loading was determined from preliminary micromechanical analyses and mixing tests, as shown in Table 1. From a consideration of the increased compressive strength and enhanced workability, a $25 \%$ addition of slag particles (in terms of the mass fraction of cement particles) was selected based on the manufacturer's recommendation and preliminary mixing tests, resulting in waterbinder ratios $(W / B)$ of $48 \%, 38 \%$, and $28 \%$.

\subsubsection{Fiber pullout tests}

A single fiber pullout test was employed to measure the interfacial properties. Figure 2(a) illustrates the specimen preparation and experimental setup. Ten specimens were cast together in a small Plexiglas mold, and then demolded after 2 days, followed by curing in water. The day prior to testing, the specimens were modified to the desired thickness, which is the fiber embedment length, $l_{\mathrm{e}}$. The fiber embedment length was chosen to be nearly $1.0 \mathrm{~mm}$ in an effort to ensure full debonding. The pullout tests were conducted on an Instron machine with the specimen configuration shown in Fig. 2(b). A $10 \mathrm{~N}$ load cell was used to measure the pullout load of the fibers with a displacement rate of $0.2 \mathrm{~mm} / \mathrm{min}$. The displacement was measured as the actuator movement. The fiber-free length was kept at a maximum of $1 \mathrm{~mm}$.

\subsubsection{Matrix fracture tests}

A wedge-splitting test (WST) method was employed to test the mortar matrix fracture toughness. The dimensions and shape of the WST specimens are shown in Fig. 3(a). An initial crack was made by inserting a steel plate (1 $\mathrm{mm}$ in this study) inside the

Table 1 Selected mix composition.

\begin{tabular}{c|c|c|c|c|c}
\hline Cement & Water & Sand & Slag & HRW & HPMC \\
\hline \hline 1.0 & 0.35 & 0.8 & 0.25 & 0.03 & 0 \\
\hline 1.0 & 0.48 & 0.8 & 0.25 & 0.02 & 0 \\
\hline 1.0 & 0.60 & 0.8 & 0.25 & 0 & 0.001 \\
\hline
\end{tabular}

*HRW : high-range water-reducing admixtrur; HPMC : hydroxy propyl methyl cellulose. All numbers are mass ratios.

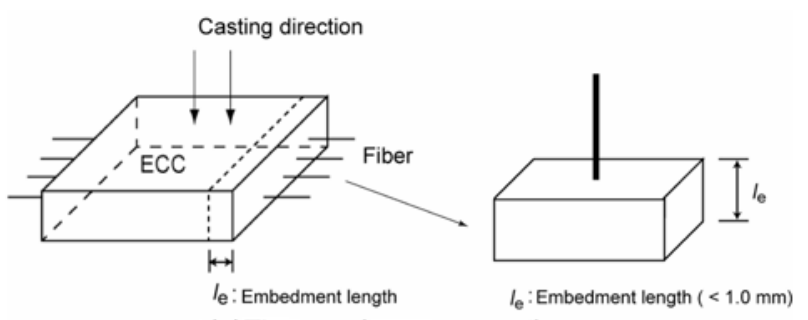

(a)The specimen preparation

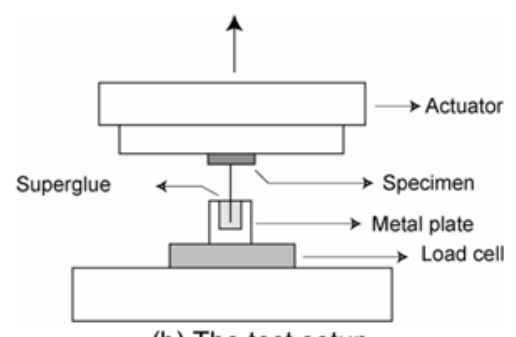

(b) The test setup

Fig. 2 Single fiber pullout test

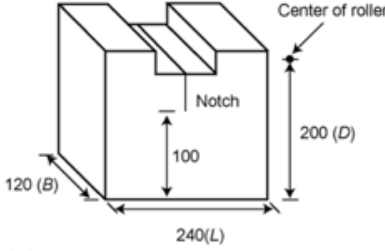

(a) The specimen geometry

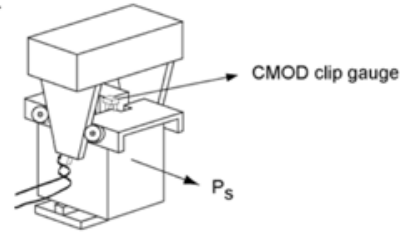

(b) The test setup
Fig. 3 Matrix fracture toughness test.

specimen during the casting process and subsequently removing the plate after 1 day. A groove was made in the upper part of the specimen for the placement of two load devices with roller bearings and the displacement gages (i.e., CMOD clip gage). The principle of the WST is schematically presented in Fig. 3(b). The actuator of the testing machine was moved so that the wedge enters between the bearings, which results in a horizontal splitting force component. The load in the vertical direction and the crack mouth opening displacement (CMOD) as well as the location of the roller axis are monitored during testing. The efficacy of this method has already been demonstrated by the authors. ${ }^{8}$ The loading speed was controlled to a constant rate $(0.06 \mathrm{~mm} / \mathrm{min})$ of CMOD increment at the location of the roller axis. The specimen was monotonically loaded to the peak load.

\subsection{Processibility tests}

\subsubsection{Deformability tests}

A regular slump cone for a conventional slump test was employed to measure the deformability of the sprayable slagECC and the self-consolidating slag-ECC. The slump test was performed at $5 \mathrm{~min}$. (self-consolidating, spraying) after mixing. This time represents the conveying time when the sprayable slagECC and the self-consolidating slag-ECC are pumped. No external means (e.g. vibration) were applied to consolidate the fresh cementitious mix. Deformability $\Gamma$ was calculated from Eq. (4) with the maximum measured diameter of the spread, $D_{1}$, and the diameter perpendicular to it, $D_{2}$.

$$
\Gamma=\frac{\left(D_{1} \times D_{2}\right)-D_{0}^{2}}{D_{0}^{2}}
$$

where, $D_{0}$ is the diameter of the slump cone $(=200 \mathrm{~mm})$.

\subsubsection{Spray tests}

A spiral pump was used for the spraying process in this study. This system is known to be particularly suitable for premixed liquids and mortar with the maximum grain size of $8 \mathrm{~mm}$. Fresh ECC mixed in a $100 \mathrm{~L}$ capacity drum mixer was pumped through this spiral pump and then down into a $35 \mathrm{~mm}$ diameter rubber hose to a spray gun, from where it was sprayed pneumatically with an air pressure of approximately $800 \mathrm{kPa}$ onto a substrate. The pumping pressure was observed during the spraying process. Once the pumping pressure reached $4 \mathrm{MPa}$, the maximum pumping pressure permitted by the pump manufacturer is aborted in all tests. The mixture design was then considered unsuitable for spraying. Sprayability was also assessed quantitatively in terms of the thickness of the fresh mixture that could be sprayed onto a substrate prior to failure under its own gravitational force, which can be termed a 'spray-on test'. The fresh mixture was 
sprayed horizontally onto a vertical surface in an attempt to obtain as large a thickness of the sprayed layer build as possible, in one continuous spraying operation.

\subsubsection{Self-consolidating tests}

The box prepared for the self-consolidating test is composed of two chambers divided by a partition in the middle. Three reinforced bars with a net spacing of $30 \mathrm{~mm}$ were positioned at the gate at the bottom of the vessel connecting the two chambers. The test was conducted by filling one of the chambers with fresh slagECC mix and subsequently removing the block at the gate. The material then flowed to the other chamber through the gate. To flow through the gate, the material had to pass through the reinforcing bars successfully without the sand or fibers separating from the matrix at the gate. The height attained by the material in the second chamber, $H$, was measured. An index for the degree of self-consolidation, $L$, was calculated by dividing this height by half of the total original height, $H_{0}$ (Eq. (5)). The index gives a value of 1 for a perfect self-consolidating performance.

$$
L=\frac{2 H}{H_{0}}
$$

\subsection{Direct tensile tests}

To verify the strain-hardening behavior of the sprayed slagECC and to compare this with the test results for the selfconsolidating slag-ECC and cast ECC (both with and without slag) specimens, a series of direct tensile tests were performed using an Instron 8506 load frame. The specimens were loaded with a constant cross head speed $(0.2 \mathrm{~mm} / \mathrm{min})$, and the loading force and elongation were measured. Two linear variable differential transducers (LVDTs) attached to both sides of the center of the tensile specimen a gage length of specimen $150 \mathrm{~mm}$ to monitor the elongation. The gage length means the distance between points A and B as shown in Fig. 4(b). To avoid fractures outside the measurement area, both ends of the specimens were made with a dog-bone shape. In addition to the tensile stress-strain curves, the ultimate tensile strength was measured, as was the ultimate tensile strain. Figure 4 shows the specimen geometry and the test setup.

\section{Results and discussions}

\subsection{Determination of basic composition of ECC produced with slag (slag-ECC)}

\subsubsection{Fiber pullout and matrix fracture tests}

The general profile of a single fiber pullout curve is illustrated in Fig. 5(a). After debonding, the pullout curve exhibits slip hardening or slip softening depending on the fiber type, fiber/ matrix bond strength, and specimen age. Based on this curve, the

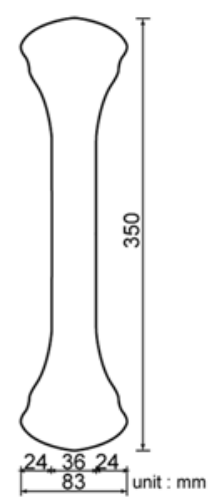

(a)The specimen geometry

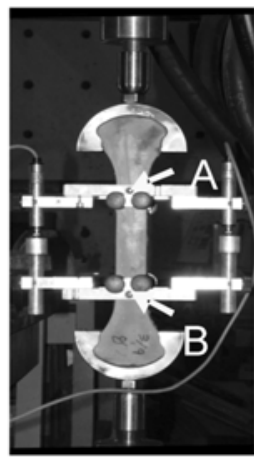

(b) The test setup
Fig. 4 Direct tensile test.

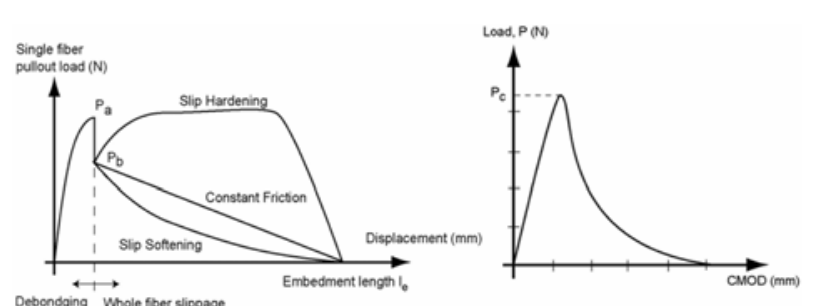

(a) A single fiber pullout test

(b) A mortar matrix fracture test

Fig. 5 Typical curves obtained.

chemical debonding energy value, $G_{d}$, is calculated from the $P_{a}$ to $P_{b}$ difference, as shown in the following Eq. (6).

$$
G_{d}=\frac{2\left(P_{a}-P_{b}\right)^{2}}{\pi^{2} E_{f} d_{f}^{3}}
$$

where, $E_{f}$ is the Young's modulus of the fiber, $P_{a}$ is the peak load during the debonding process, $P_{b}$ is the peak load during the pullout process, and $d_{f}$ is the fiber diameter. ${ }^{9}$ From the $P_{b}$ value, the frictional bond strength $\tau_{0}$ at the onset of fiber slippage is calculated as follows

$$
\tau_{0}=\frac{P_{b}}{\pi d_{f} l_{e}}
$$

A typical load-CMOD curve is shown in Fig. 5(b), from which the critical CMOD $\left(\mathrm{CMOD}_{c}\right)$, and critical peak load, $P_{c}$ are obtained. The fracture toughness, $K_{I c}\left(=K_{m}\right)$, and the elastic modulus of the matrix, $E_{c}$, were calculated from the load-CMOD curves. Details of the empirical calculations can be found in a study by Murakami and Zhao. ${ }^{10,11}$ Finally, $J_{\text {tip }}$ through the use of Eq. (2) is calculated.

Tables 2 and 3 display the results obtained from the tests. As compared in these tables, there is no significant difference in $G_{d}$, independent of the addition of slag particles. This is most likely

Table 2 Test results (matrix with slag particles).

\begin{tabular}{c|c|c|c|c|c|c|c|c|c}
\hline & \multicolumn{3}{|c|}{$W / B=48 \%(W / C=60 \%)$} & \multicolumn{3}{c|}{$W / B=38 \%(W / C=48 \%)$} & \multicolumn{3}{c}{$W / B=28 \%(W / C=35 \%)$} \\
\hline \hline Age & $\tau_{o}(\mathrm{MPa})$ & $G_{d}\left(\mathrm{~J} / \mathrm{m}^{2}\right)$ & $J_{\text {tip }}\left(\mathrm{J} / \mathrm{m}^{2}\right)$ & $\tau_{o}(\mathrm{MPa})$ & $G_{d}\left(\mathrm{~J} / \mathrm{m}^{2}\right)$ & $J_{\text {tip }}\left(\mathrm{J} / \mathrm{m}^{2}\right)$ & $\tau_{o}(\mathrm{MPa})$ & $G_{d}\left(\mathrm{~J} / \mathrm{m}^{2}\right)$ & $J_{\text {tip }}\left(\mathrm{J} / \mathrm{m}^{2}\right)$ \\
\hline 7d & 1.39 & 1.77 & 2.02 & 1.42 & 1.79 & 3.28 & 1.47 & 1.81 & 6.43 \\
\hline 14d & 1.43 & 1.83 & 2.79 & 1.50 & 1.87 & 4.63 & 1.54 & 1.87 & 8.92 \\
\hline 28d & 1.65 & 1.88 & 3.23 & 1.85 & 1.83 & 5.42 & 1.94 & 1.86 & 10.04 \\
\hline
\end{tabular}


Table 3 Test results (matrix without slag particles).

\begin{tabular}{c|c|c|c|c|c|c|c|c|c}
\hline & \multicolumn{3}{|c|}{$W / C=60 \%$} & \multicolumn{3}{c|}{$W / C=48 \%$} & \multicolumn{3}{c}{$W / C=35 \%$} \\
\hline \hline Age & $\tau_{o}(\mathrm{MPa})$ & $G_{d}\left(\mathrm{~J} / \mathrm{m}^{2}\right)$ & $J_{\text {tip }}\left(\mathrm{J} / \mathrm{m}^{2}\right)$ & $\tau_{o}(\mathrm{MPa})$ & $G_{d}\left(\mathrm{~J} / \mathrm{m}^{2}\right)$ & $J_{\text {tip }}\left(\mathrm{J} / \mathrm{m}^{2}\right)$ & $\tau_{o}(\mathrm{MPa})$ & $G_{d}\left(\mathrm{~J} / \mathrm{m}^{2}\right)$ & $J_{\text {tip }}\left(\mathrm{J} / \mathrm{m}^{2}\right)$ \\
\hline 7d & 1.30 & 1.80 & 1.68 & 1.40 & 1.92 & 2.85 & 1.49 & 1.80 & 5.49 \\
\hline 14d & 1.36 & 1.81 & 2.35 & 1.49 & 1.84 & 3.81 & 1.53 & 1.84 & 7.26 \\
\hline 28d & 1.62 & 1.82 & 2.67 & 1.82 & 1.85 & 4.53 & 1.90 & 1.70 & 8.59 \\
\hline
\end{tabular}

because the PVA fiber, a hydrophilic fiber, shows a strong chemical bond with the fresh cement matrix, of which the strength is principally determined by the chemical structures developed between the fiber surface and cement matrix. These chemical structures were not altered by the water content and thus the chemical bond is independent of the matrix $W / B .{ }^{9}$ In contrast, the addition of slag particles results in a slight increase (within $10 \%$ ) of $\tau_{0}$ at the same $\mathrm{W} / \mathrm{C}$. This is due to the decreased porosity in the interfacial zone with the decrease of $W / B$, which can lead to an increase of the fiber-matrix contact surface, resulting in a higher friction bond.

The effects of the $W / C$ and the addition of slag particles on $J_{t i p}$ are illustrated in Fig. 6 as a function of the age of the matrix. The results indicate that the $J_{t i p}$ value increases with the addition of slag particles at an identical $W / C$. This is primarily due to the enhanced matrix strength caused by the presence of slag particles. Slag particles are most commonly activated by the hydration product of Portland cement, where calcium hydroxide formed during hydration is the principal activator. Slag hydration products lead to decreased porosity in the matrix, resulting in enhanced matrix strength and toughness. Higher matrix toughness may be detrimental to achieving the desired mechanical properties, as it reduces the margin to develop multiple cracking in terms of toughness ratio. However, it should be noted that at an identical $W / B$ ratio, the mix designed with slag particles exhibits lower $J_{t i p}$, compared to this value of the mix without slag. This is almost certainly due to the retarded cementitious reactions of the slag, which consume part of the calcium hydroxide formed during the earlier stages of hydration. This indicates that the use of slag particles should be helpful for achieving strain-hardening behavior, as lower a $J_{t i p}$ value provides better chances for multiple cracking in the composite.

\subsubsection{Fiber bridging analysis}

To obtain the toughness ratio $\left(J_{b}^{\prime} / J_{t i p}\right)$, a fiber bridging analysis was performed on the composites that were reinforced with $2 \%$ PVA fibers in a volume fraction based on the micromechanical

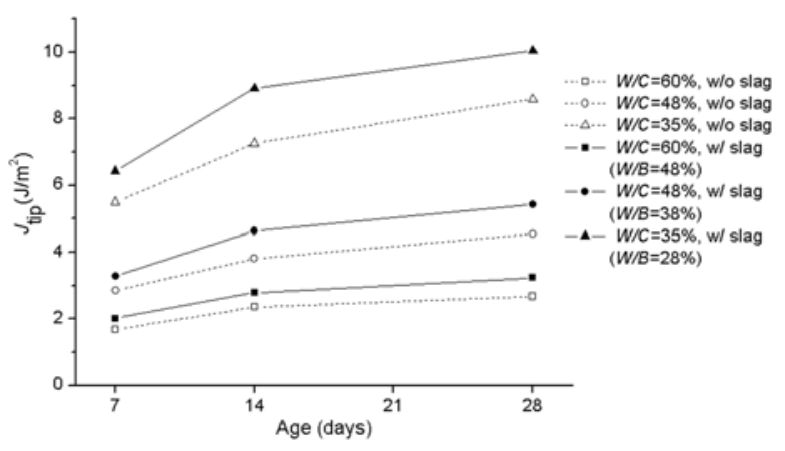

Fig. 6 Crack tip toughness $J_{t i p}$ as a function of the matrix age for the effects of W/C and the addition of slag particles. principle. ${ }^{2}$ The toughness ratios calculated from these analyses are displayed in Fig. 7. As shown in Fig. 7, all mixes sufficiently meet the steady state cracking requirement, specifically $\left(J_{b}{ }^{\prime}\right.$ $\left.J_{\text {tip }}\right)=1$. However, from an experimental correlation between the intensity of multiple crack saturation and the toughness ratio $\left(J_{b}{ }^{\prime}\right)$ $J_{\text {tip }}$ ) of the composite, Kanda and Li estimated that a toughness ratio value equal to 3 is necessary to achieve saturated multiple cracking. ${ }^{12}$ Based on their investigation, it was estimated that mixes of $35 \% \mathrm{~W} / \mathrm{C}$ both with and without slag particles are not appropriate for obtaining saturated multiple cracking. In addition, observations in the present set of experiments appear to suggest that the mixes of $35 \% \mathrm{~W} / \mathrm{C}$ are scarcely workable with the use of an ordinary mixing system. In terms of the toughness ratio and processibility, a $W / C$ ratio higher than $35 \%$ is preferable in practice.

\subsubsection{Optimized range of basic composition}

For the processing design of fresh slag-ECC mixture, an appropriate range of ingredient loading from extensive micromechanical analyses and experiments was predetermined. It was disclosed that a water-cement ratio $(W / C)$ ranging from $48 \%$ to $60 \%$ with a sand-cement ratio $(S / C)$ ratio of $80 \%$ would be appropriate to achieve satisfactory mortar matrix properties. To demonstrate an optimal $W / C$ ratio, direct tensile tests were performed on the composites with $W / C$ s of $48 \%$ and $60 \%$ (both with and without slag) at 28 days, respectively. The direct tensile test is considered to be the most accurate and effective method to confirm the strain-hardening behavior of a composite, as quasi-brittle FRC is potentially able to show apparent strainhardening behavior under flexural loading, depending on the specimen geometry. The test results in terms of typical stressstrain curves are presented in section 4.3.

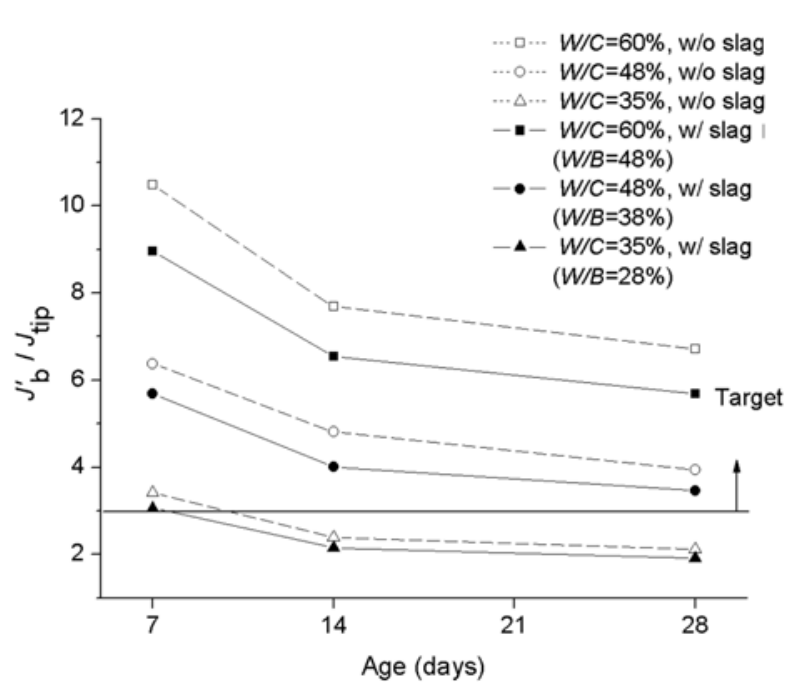

Fig. 7 The toughness ratio as a function of the composite age for the effects of W/C and the addition of slag particles. 


\subsection{Controlled processibility of slag-ECC}

\subsubsection{Deformability of slag-ECC}

Based on previous studies ${ }^{13}$, the mixture proportion of ECC, suitable for spraying and self-consolidating processes, was optimized as given in Table 4. To obtain the deformability of slag-ECC, a deformability test was performed with a conventional slump cone $\left(D_{1}=370 \mathrm{~mm}, D_{2}=350 \mathrm{~mm}\right.$; sprayable slag-ECC, $D_{1}=670 \mathrm{~mm}, D_{2}=610 \mathrm{~mm}$; self-consolidating slag-ECC). The deformability of the fresh sprayable slag-ECC mix and selfconsolidating slag-ECC mix, $\Gamma$, were 2.2 and 9.2, respectively. These values were determined based on an averaged result of two deformability tests. The degree of self-consolidating ECC is comparable to the deformability of self-consolidating concrete, ranging from 8 to $12 .{ }^{14,15}$ Figure 8 shows the deformability of the sprayed slag-ECC and self-consolidating slag-ECC in one of the deformability tests. The slag-ECCs shown in Fig. 8 are expected to achieve diverse types of processing.

\subsubsection{Spraying processibility}

To verify the sprayability of the fresh slag-ECC mixture, sprayon tests were performed. No excess pumping pressure was observed during the spray tests, indicating superior pumpability when compared to the pressure observed in a previous study. ${ }^{2}$ This may be due to the fact that slag particles were introduced, and were expected to enhance the workability and pumpability. It may as well be attributed to the oxidized grain surface of the slag. As shown in Fig. 9, the sprayability was assessed quantitatively to obtain as large a thickness of sprayed slag-ECC as possible. A maximum thickness of $50 \mathrm{~mm}$ was obtained by spraying onto a vertical surface in a continuous process. This value of $50 \mathrm{~mm}$ is comparable with the typically applied thickness of shotcrete layer, approximately $50 \mathrm{~mm}$, in repair work. ${ }^{2}$

\subsubsection{Self-consolidating processibility}

To demonstrate the self-consolidating ability of the fresh mix, a self-consolidating test was performed with a box $(H=125 \mathrm{~mm}$; Fig. 10). The self-consolidation index, $L$, of the fresh slag-ECC mix was 0.83 . The fresh mix exhibits a smooth surface, while the reinforcing bars placed under the partition of the box tend to imprint their shapes onto the surface of the fresh ECC mix. This demonstrates the satisfactory self-leveling performance of the fresh

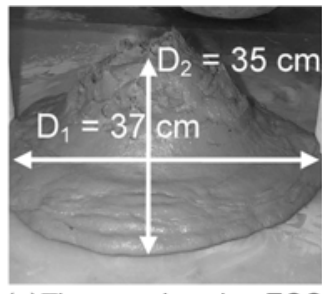

(a) The spraying slag-ECC

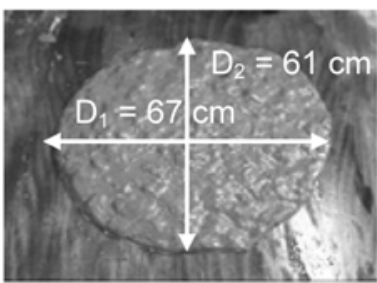

(b) The self-consolidating slag-ECC
Fig. 8 Deformability tests.

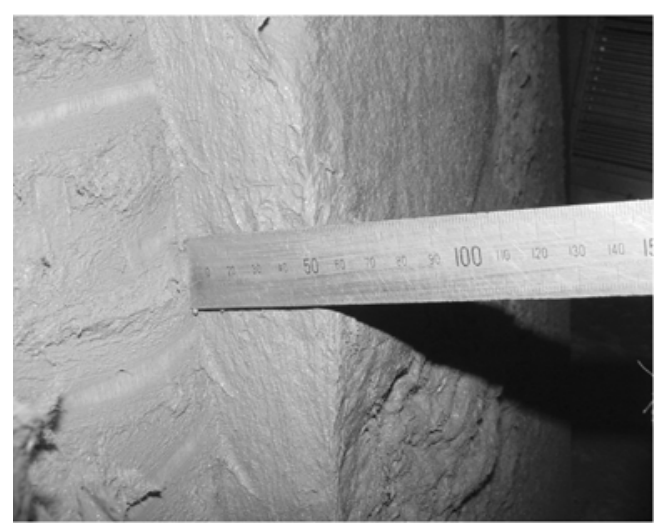

Fig. 9 Spray-on test to a vertical surface for a $50 \mathrm{~mm}$ thickness of sprayed ECC layer.

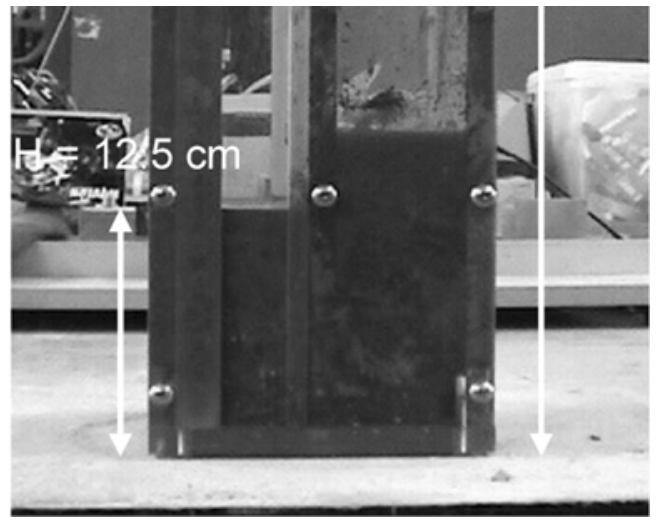

Fig. 10 Demonstration of the self-consolidating property of the fresh ECC mix.

slag-ECC mixture. In addition, the value of the self-consolidation index is comparable to that of self-consolidating concrete, ranging from 0.73 to $1 . .^{14,15}$

\subsection{Tensile performance demonstration}

Uniaxial tensile tests were performed on cast, sprayed and selfconsolidated specimens of the slag-ECC in order to confirm the ductile strain-hardening performance of the hardened composite. The stress-strain curves are presented in Fig. 11. All specimens exhibit apparent multiple cracking patterns accompanying pseudo strain-hardening behavior with strain capacities ranging from 1.0 to nearly $3.6 \%$. This ductility remains much higher than that in normal concrete and conventional FRC composites. However, the mixes of $W / C=48 \%$ show relatively lower strain capacity when compared to the ductility of the mixes of $W / C=60 \%$. It appears that the toughness ratio of the mixes with a $W / C=48 \%$ was very close to the required $\left(J_{b}^{\prime} / J_{\text {tip }}=3\right)$, thus a sufficient margin for obtaining saturated multiple cracking cannot be provided. However, at an identical water-binder ratio, slag-ECC with a $W /$ $B=48 \%$ exhibits a much higher strain capacity when compared

Table 4 Composition of sprayable ECC and self-consolidating ECC.

\begin{tabular}{c|c|c|c|c|c|c|c}
\hline Mix & $W / C$ & $S / C$ & $\mathrm{~V}_{\mathrm{f}}(\mathrm{vol}$.) & Slag/C & HRW/C (\%) & HPMC/C (\%) & CA/C (\%) \\
\hline \hline Spraying & \multirow{2}{*}{0.55} & \multirow{2}{*}{0.8} & \multirow{2}{*}{0.02} & \multirow{2}{*}{0.25} & 0.8 & 0.3 & 5 \\
\cline { 1 - 5 } Self-consolidating & & & & & 3.0 & 0.2 & - \\
\hline
\end{tabular}

$\mathrm{C}$ : cement, W : water, $\mathrm{S}:$ sand, Slag : ground granulated blast furnace slag, HPMC : hydroxy propyl methyl cellulose, HRW : high-range waterreducing admixture, $\mathrm{CA}$ : calcium aluminate cement, $\mathrm{V}_{\mathrm{f}}$ : fiber volume fraction, - : not available. All numbers are mass ratios except for $\mathrm{V}_{\mathrm{f}}$. 


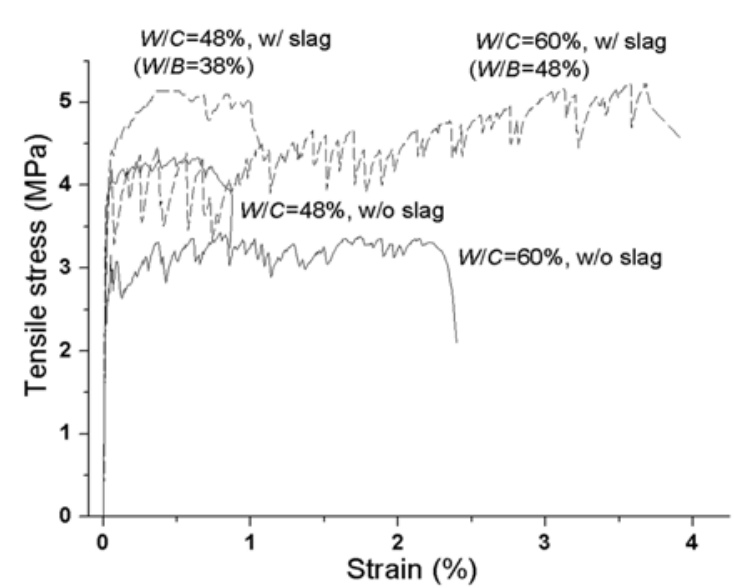

(a) Cast ECC

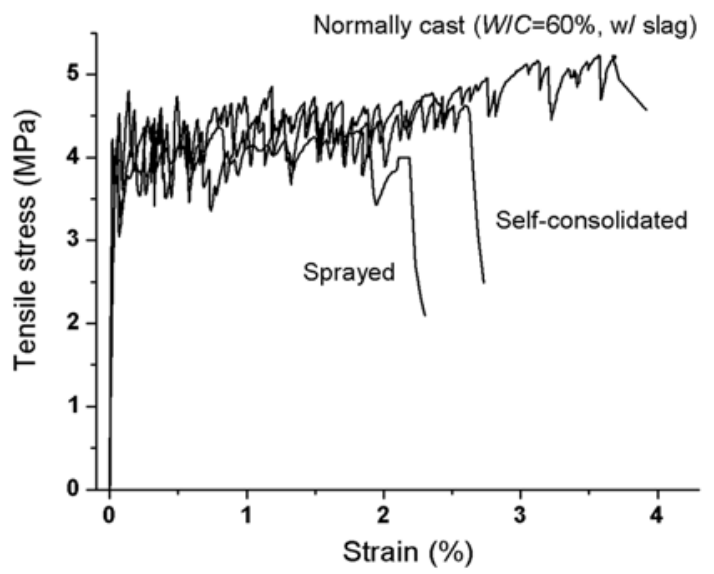

(b) Sprayed and self-consolidating slag-ECC compared with cast ECC at 28 days

Fig. 11 Comparison of the uniaxial tensile stress vs. the strain curves.

to the composite with a $W / C=48 \%$. This is mainly due to the fact that slag-ECC with a $W / B=48 \%$ has a $40 \%$ higher toughness ratio compared to that of the $W / C=48 \%$ mix, as shown in Fig. 7, which indicates that the $W / B=48 \%$ mix has a sufficient margin for obtaining saturated multiple cracking, as previously described in section 4.1.2.

The mixes of $W / C=60 \%$ exhibit a strain capacity that is more than $2 \%$ higher. Moreover, the addition of slag particles $(W /$ $B=48 \%$ mix with slag) leads to an enhanced tensile strain capacity $(\sim 3.6 \%)$ accompanied by greater composite strength ( $44 \mathrm{MPa}$ in compressive strength) despite the smaller margin of toughness ratio due to slightly higher frictional bond and matrix toughness when compared to the composite without slag. Both the ductility and strength of the composite with slag were measured to be significantly higher than those of the composite without slag (strain capacity of approximately $2.4 \%$ and compressive strength of approximately $26 \mathrm{MPa}$ ). As previously expected, this likely resulted from the presence of slag particles. The slag particles contribute to improving fiber dispersion, which is associated with enhanced workability attributed to the electrical double layer formed by the oxidized grain surface. This result suggests that, within the limited slag dosage employed in the present experiments, the contribution of slag particles to the workability outweighs the side-effect of decreased potential of saturated multiple cracking.

The ductility of the sprayed and self-consolidating ECC is comparable with that of slag-ECC specimens cast with external consolidation. Such a consistent material property is likely due to the sufficient compaction and good fiber dispersion during the spraying and self-consolidating processes. It was reconfirmed that the slag-ECC was properly processed by spraying and selfconsolidating.

\section{Conclusions}

A basic slag-ECC composition with satisfactory tensile behavior was developed by employing micromechanical tools and the steady state cracking theory. With these parameters, diverse types of processing (i.e., self-consolidating and spray processing) were developed by optimizing the dosage of admixtures. To demonstrate pseudo strain-hardening behavior of the slag-ECC, direct tensile tests were conducted. The following conclusions can be drawn from the experimental results:

1) An optimized range of the basic composition with slag particles was obtained. This was determined based on micromechanics and the steady-state cracking theory. A micromechanical analysis was initially performed to properly select the watercement ratio, and the basic matrix proportion range was then determined based on the analysis results and workability considerations, including the desirable fiber dispersion without segregation.

2) Both ductility ( $\sim 3.6 \%$ for the tensile strain capacity) and strength ( $44 \mathrm{MPa}$ for the compressive strength) of the slagECC were measured to be significantly higher than these values for the ECC without slag. The ECC mix designed with slag particles exhibited a lower $J_{t i p}$ value at an identical $W / B$ ratio, compared to that of the mix without slag. Therefore, slag-ECC demonstrated a higher toughness ratio when compared to ECC (without slag) at an identical $W / B$ ratio. This indicates that the use of slag particles should be helpful for achieving strain-hardening behavior, as the higher toughness ratio provides a better chance for multiple cracking in the composite.

3) Although the toughness ratio decreases with the addition of slag particles at an identical $W / C(60 \%)$, the tensile strain capacity of slag-ECC is enhanced. This results primarily as the slag particles contribute to improving the fiber dispersion, which is associated with enhanced workability attributed to the electrical double layer formed by the oxidized grain surface. This result also suggests that, within the limited slag dosage employed in the present experiments, the contribution of slag particles to the workability outweighs detrimental side-effects (higher $J_{\text {tip }}$ ) of the decreased potential of saturated multiple cracking.

4) The performance of the desired processing (self-consolidating or spray processing), while retaining the ductile material properties, was obtained by adopting control of the rheological modulation. The rheological properties of the composite were controlled by modulating the dosage of the admixtures. Moreover, the ductility of the sprayed and self-consolidating slag-ECC was comparable with that of ECC specimens ordinarily cast with external consolidation.

\section{Acknowledgements}

\author{
Research funding from KICTEP at KAIST (A06-04) is \\ gratefully acknowledged.
}




\section{References}

1. Li, V. C., "Reflections on the Research and Development of ECC," Proceedings of the JCI Int'l Workshop on Ductile Fiber Reinforced Cementitious Composites Application and Evaluation (DRFCC'2002), Takayama, Japan, Oct. 2002, pp.1 21.

2. Kim, Y. Y., Kong, H. J., and Li, V.C., "Design of Engineered Cementitious Composite Suitable for Wet-mixture Shotcreting," ACI Materials Journal, Vol.100, No.6, 2003, pp.511 518.

3. Li, V. C. and Leung, C. K. Y., "Steady State and Multiple Cracking of Short Random Fiber Composites," Journal of Engineering Mechanics, ASCE, Vol.188, No.11, 1992, pp.2246 2264.

4. Park, C. K., Noh, M. H., and Park, T. H., "Rheological Properties of Cementitious Materials Containing Mineral Admixtures," Cement and Concrete Research, Vol.35, No.5, 2005, pp.842 849.

5. N. Feng, Y. and Shi, T. Hao, "Influence of Ultra-Fine Powder on the Fluidity and Strength of Cement Paste," Advances in Cement Research, Vol.12, No.3, 2000, pp.89 95.

6. Y. Shi, I. and Matsui, Y. Guo, "A Study on the Effect of Fine Mineral Powders with Distinct Vitreous Contents on the Fluidity and Rheological Properties of Concrete," Cement and Concrete Research, Vol.34, No.8, 2004, pp.1381 1387.

7. Kong, H. J., Bike, S., and Li, V. C., "Constitutive Rheological Control to Develop a Self-Consolidating Engineered Cementitious Composite Reinforced with Hydrophilic Poly(Vinyl alcohol) Fibers," Cement and Concrete Composites, Vol.25, No.3, 2003, pp.333 341.
8. Kim, J. K. and Kim, Y. Y., "Fatigue Crack Growth of High Strength Concrete in Wedge Splitting Test," Cement and Concrete Research, Vol.29, No.5, 1999, pp.705 712.

9. Kanda, T. and Li, V. C., "Interface Property and Apparent Strength of High-strength Hydrophilic Fiber in Cement Matrix," ASCE Journal of Materials in Civil Engineering, Vol.10, No.1, 1998, pp.5 13.

10. Murakami, Y., et al., Stress Intensity Factors Handbook, Pergamon Press, New York, 1987.

11. Zhao, G., Jiao, H., and Xu, S., "Study on Fracture Behavior with Wedge Splitting Test Method," Fracture Processes in Concrete, Rock and Ceramics, E\&F.N. Spon, London, 1991, pp.789 798.

12. Kanda, T. and Li, V. C., "A New Micromechanics Design Theory for Pseudo Strain-Hardening Cementitious Composite," Journal of Engineering Mechanics, ASCE, Vol.125, No.4, 1999, pp.373 381.

13. Kim, Y. Y., Kim, J. S., Kim, J. K., and G. J. Ha, "Processibility of High Ductile Fiber-Reinforced ECCs," Proceedings of the Conference of Korea Concrete Institute (KCI), Vol.17, No.1, 2005, pp.313 316.

14. Okamura H. and Ozawa K., "Mix-design for Self-compacting Concrete," Concr. Lib., JSCE, Vol.25, 1995, pp.107 120.

15. Nagamoto N. and Ozawa K., "Mixture Proportions of Self-compacting High-performance Concrete. In: High-performance Concrete: Design, Materials, and Advances in Concrete Technology," ACI International, SP-172, 1999, pp.623 666. 